\title{
Emergence of Specialization from Global Optimizing Evolution in a Multi-agent System
}

\author{
Lei Chai, Jiawei Chen, Zhangang Han, Zengru Di, and Ying Fan* \\ Center for Complexity Research, \\ Beijing Normal University, Beijing, 100875, P.R. of China \\ Institute of Social Development and Public Policy, \\ Beijing Normal University, Beijing, 100875, P.R. of China \\ \{1chai, jwchen, zhan, zdi, yfan\} @bnu. edu.cn \\ http://www.springer.com/lncs
}

\begin{abstract}
A Markov chain model is proposed to describe the evolutionary dynamics of a multi-agent system. Many individual agents search for and exploit resources to get global optimization in an environment without complete information. With the selection acting on agent specialization at the level of system and under the condition of increasing returns, agent specialization emerges as the result of a long-term optimizing evolution.
\end{abstract}

Keywords: agent specialization, evolutionary dynamics, multi-agent system.

\section{Introduction}

Among novel and numerous varieties of collective activities performed by social insect societies, such as all ants and termites, and some species of bees and wasps 1, division of labor is a typical example which is classically viewed as an evolving process led by mutation and selection, and also has emergent properties of social systems. A lot of work has been done to study the formation of division of labor and the mechanism for tasks allocation 2345. In this paper, multiagent system simulation has been applied and a framework of pattern formation has been borrowed to study specialization phenomena in biological, social and economic systems.

The emergence of collective behaviors in multi-agent systems has become an interesting area of complexity research 6/7/891011. In a distributed multiagent system, be it natural or social, agents do not have complete information about the environment where they live, and they have to actively interact with others to reach collective or individual optimization. Even though the interactions among agents may be simple and local, they can lead to complex dynamics at the global scale. Studies of computational ecology have shown that when the agents make choices with imperfect information, the dynamics can give rise to nonlinear oscillations, clustered volatilities and chaos that drive the system

\footnotetext{
^ Corresponding author.
} 
far from optimality [1213]. Moreover, when the agents in the system have a hierarchical organization that determines the task allocations, the system as a whole will be able to manage complex and various changing environments 214. Holland has argued that complexity arises from the self-adaptive properties of individual agents [15] and this approach has been applied to biological ecosystems and economic systems [16]17/18].

Evolutionary processes and principles are helpful to understand the mechanisms behind the formation of specialization. As to a long-term evolution, selection acting on agent specialization must take place at the level of the colony. Some colonies survive and reproduce more than others because they have a division of labor that is better adapted to a particular environment [19]. Based on our previous multi-agent simulation model of agent division [20, this paper presents a Markov Chain model that describes a system of many individual agents that search for and exploit resources to get global optimization in an environment where they interact locally. Under the condition of increasing returns, specialization is unfailingly the result from the global optimizing evolution under certain initial conditions.

The presentation is organized into three major parts. In Section 2, the model and the results of static analysis are presented. Section 3 gives the Markov chain model describing the evolutionary dynamics of the system. The final steady state shows the specialization of the agents. The results are well consistent with that of simulation model. In Section 4, we provide a summary of our results and a brief discussion of some unresolved issues.

\section{The Model and Static Analysis}

Consider a system with $M$ individual agents. Every agent is an autonomous entity which searches for and exploits resources in a given environment. There is complete information transfer among agents. Each agent knows the information about the resource distribution which is specified by every other agent's forage. For simplicity and without loss any generality, we introduce a simple, non-spatial model of evolutionary dynamics to obtain some mathematical analytical results.

\subsection{Optimal Behavior for Solitary Agent}

First, let's discuss the problem for a solitary agent to deal with an uncertain environment.

The live space for the agent is composed by the samples of resources valued $F_{0}$ and 0 . In the beginning of every period of time, the agent can choose to search for new resources with probability $q$ or to take the situation of last period with probability $1-q$. When the agent determines to search new resource, it will get the resource $F_{0}$ with probability $P$. Then its product is $(1-q) F_{0}$. The cost for the search is $(1-q) C$, where the factor $1-q$ describes the effect of learning by 
doing[21]. When the agent determines to take the last situation, if the last value of the resource is $F_{t-1}$, the value of this period is

$$
F_{t}=\frac{1}{a} F_{t-1}, a>1
$$

where $a$ stands for the depreciation of resources and $t$ denotes the generation of the system's evolution. The goal of the agent is to maximize the total net returns for $N$ periods, which is determined by each agent's parameter $q$ for a given distribution of resources that is described by probability $P$.

In the beginning of searching and exploiting process noted as period 0 , the agent randomly searches. For this period, the expected return is $E_{0}=(1-q)$ $\left(P F_{0}-C\right)$. For period 1, the agent can choose to search for new resources with probability $q$. The corresponding expected benefit is $E_{0}$. Another choice is to stay in the situation of last period (with probability $1-q$ ). The corresponding expected return is $P F_{0} / a$. So the expected return of period 1 is

$$
E_{1}=(1-q)\left(q E_{0}+(1-q) \frac{1}{a} P F_{0}\right)
$$

Let $x=(1-q) / a$. From the similar analysis, the expected benefit of period $n$ can be written as:

$$
E_{n}=(1-q)\left(q E_{0}+P F_{0}\left(x^{n}+q \frac{x\left(1-x^{n-1}\right)}{1-x}\right)\right)
$$

Hence the total expected returns from period 0 to period $N$ is

$$
\begin{aligned}
& W=\sum_{n=0}^{N} E_{n}=(1+N)(1-q) q E_{0}+P F_{0}(1-q) \sum_{n=0}^{N}\left(x^{n}+q \frac{x\left(1-x^{n-1}\right)}{1-x}\right) \\
& =(1+N)(1-q) q E_{0}+P F_{0}(1-q)\left[(1+N) \frac{q x}{1-x}+\left(1-\frac{q}{1-x}\right) \frac{1+x^{N}}{1-x}\right]
\end{aligned}
$$

From the above result, we can obtain the optimal point $q_{M}$ and corresponding maximum total expected returns $W$ for given set of parameters.

\subsection{Optimal Division of Agents for a Colony}

Now we turn to the colony of $M$ agents. Each agent is characterized by a parameter $q_{i}$, which determines the searching probability of the agent. As discussed in the above solitary agent case, we assume that every searching agent finds resources $F_{0}$ with probability $P$. Assume there is complete information between agents. If any agent has found a resource $F_{0}$, all the others will go and exploit it. Then the product of the system is $\sum_{i=1}^{M}\left(1-q_{i}\right) F_{0}$. The searching cost for each agent is $\left(1-q_{i}\right) C$.

Let's compare the results on the total returns of distributed $q_{i}$ with complete specialization. Hence at every time period, there will be $\sum_{i=1}^{M} q_{i}=m$ agents searching for new resources. The gross probability of finding at least one new 
resource $F_{0}$ is the same with when there is $m$ agents specialized in searching. We denote this gross probability as $P_{r}$. For a colony with distributed $q_{i}$, the expected product of the first period is $R_{D}=\sum_{i=1}^{M}\left(1-q_{i}\right) F_{0} P_{r}=M F_{0} P_{r}-$ $F_{0} P_{r} \sum_{i=1}^{M} q_{i}=F_{0} P_{r}(M-m)$. The cost for searching is $C_{D}=\sum_{i=1}^{M} q_{i}\left(1-q_{i}\right) C=$ $m C-C \sum_{i=1}^{M} q_{i}^{2}$. With $q_{i} \leq 1, \sum_{i=1}^{M} q_{i}^{2}$ is usually less than $m$. So we usually have $C_{D}>0$. But for the colony with specialized agents, although the expected product of the first period is the same: $R_{S}=\sum_{i=1}^{M-m} F_{0} P_{r}=F_{0} P(M-m)=R_{D}$, hence the products of following generations are also the same, and the cost for search is 0 . So the net return of a specialized system is bigger than that of the distributed one.

Assuming that there are $m$ agents specialized in searching in the colony, all the others specialized in exploiting the resource. In each period, If any searching agent has the probability $P$ to find the resource $F_{0}$, then the probability for $m$ agents at least find one resource $F_{0}$ is $P_{r}=1-(1-P)^{m}$. So the expected returns of the colony for every period are:

$$
\begin{aligned}
& E_{0}=P_{r}(M-m) F_{0} \\
& E_{1}=P_{r}(M-m) F_{0}+P_{r}\left(1-P_{r}\right) \frac{1}{A}(M-m) F_{0} \\
& E_{2}=P_{r}(M-m) F_{0}+P_{r}\left(1-P_{r}\right) \frac{1}{A}(M-m) F_{0}+P_{r}\left(1-P_{r}\right)^{2} \frac{1}{A^{2}}(M-m) F_{0}
\end{aligned}
$$

Let $x=\left(1-P_{r}\right) / A$, where parameter $A>1$ is also related to the diminishing of resource under agents' exploiting. The return of $n t h$ period can be written as

$$
E_{n}=E_{0}\left(\frac{1-x^{n}}{1-x}\right)
$$

So the total returns of $N$ periods is

$$
\begin{array}{r}
W\left(\left\{N_{j}\right\}\right)=\sum_{n=0}^{N-1} E_{0} \frac{1-x^{n}}{1-x}=\frac{E_{0}}{1-x}\left(N-x\left(\frac{1-x^{N}}{1-x}\right)\right) \\
=\frac{P_{r}(M-m) F_{0}}{1-x}\left(N-x\left(\frac{1-x^{N}}{1-x}\right)\right)
\end{array}
$$

By equation 5, it is found that $m_{0}$ is sensitive to the probability $P$ (as shown in Figure 1). The results are in good agreement with previous simulation results.

\section{Markov Chain Model for the Evolutionary Dynamics}

We assume the character space of the agents has $k+1$ states corresponding to the searching probabilities described by parameter $q_{j}, j=0,1, \ldots, k$, with $q_{0}=0$ and $q_{k}=1$. The distribution of agents in every state describes the situation of the colony in macroscopic level. Let's denote this distribution as $\left\{N_{j}, j=0,1, \ldots, k\right\}$, and we have $\sum_{j=0}^{k} N_{j}=M$. For generality $N_{j}$ is a positive real number instead 


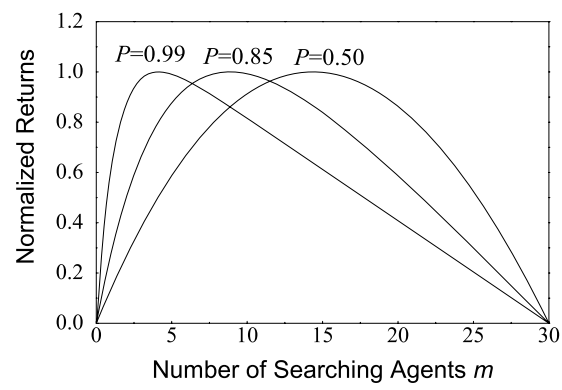

Fig. 1. Returns (normalized) as a function of number of searching agents $m$. Except parameter $P$ (labeled above the corresponding curve), all the other parameters for each curve are the same.

of a positive integer. From the results in the above discussion, the total product $W\left\{N_{j}\right\}$ and total cost $C_{D}\left\{N_{j}\right\}$ of the colony in one generation with $N$ periods is

$$
\begin{gathered}
W\left(\left\{N_{j}\right\}\right)=\frac{P_{r}\left(M-\sum_{j=0}^{k} N_{j} q_{j}\right) F_{0}}{1-x}\left(N-x\left(\frac{1-x^{N}}{1-x}\right)\right) \\
C_{D}\left(\left\{N_{j}\right\}\right)=N \sum_{j=0}^{k} N_{j} q_{j}\left(1-q_{j}\right) C
\end{gathered}
$$

where $x=\left(1-P_{r}\right) / A, P_{r}=1-(1-P)^{m}$, and $m=\sum_{j=0}^{k} N_{j} q_{j}$. The total return of the system in one generation is $R\left(\left\{N_{j}\right\}\right)=W\left(\left\{N_{j}\right\}\right)-C_{D}\left(\left\{N_{j}\right\}\right)$.

Within the evolution process between two generations, the state of every agent will transit among all the $k+1$ states. In the simulation model, we only make it possible for the transition between the nearest neighbors. A Markov chain process can describe the genetic variation and natural selection model. The dynamical behavior of this Markov chain is determined by following equations:

$$
\begin{array}{r}
N_{j}(t+1)=N_{j}(t)+P(j+1 \rightarrow j) N_{j+1}(t)+P(j-1 \rightarrow j) N_{j-1}(t) \\
-P(j \rightarrow j-1) N_{j}(t)-P(j \rightarrow j+1) N_{j}(t)
\end{array}
$$

For $j=0$ and $j=k$ we have

$$
\begin{array}{r}
N_{0}(t+1)=N_{0}(t)+P(1 \rightarrow 0) N_{1}(t)-P(0 \rightarrow 1) N_{0}(t) \\
N_{k}(t+1)=N_{k}(t)+P(k-1 \rightarrow k) N_{k-1}(t)-P(k \rightarrow k-1) N_{k}(t)
\end{array}
$$

where $P(i \rightarrow j)$ is the probability for an agent to transit from state $i$ to state $j$, and it is determined by the global optimization. Corresponding to the natural selection process described in the simulation model, the transition probability can be written as:

$$
P(i \rightarrow j)=\frac{1}{2} \mu\left[5+3 \operatorname{sgn}\left(R\left(N_{i}-1, N_{j}+1\right)-R\left(N_{i}, N_{j}\right)\right)\right]
$$



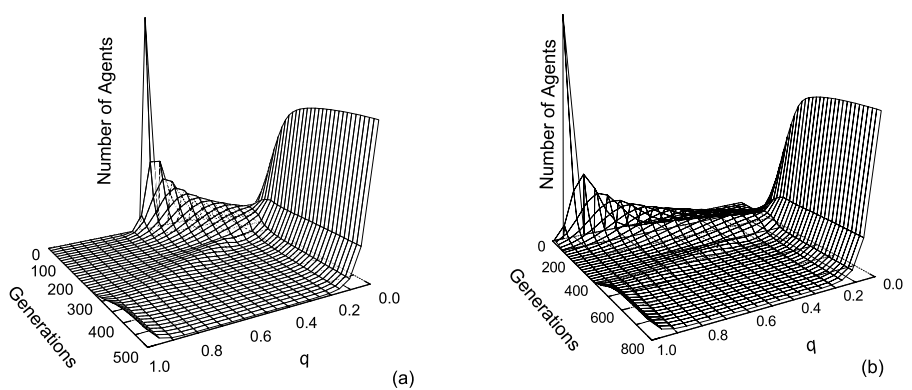

(a)

(b)

Fig. 2. Evolution of the distribution of agents. The results are qualitative similar to the computer simulations.

where $\mu$ is a parameter related to the probability of mutation. From the above equations, we can get the results of the optimal evolution of the system from any given initial conditions, which is shown in Figure 2, The Markov chain model is carried out under the following parameters. $M=30$ agents form a colony and want to get global optimization in an uncertainty environment. The probability for every searching agent to find resource $F_{0}$ is $P=0.7$. The value of the resource is $F_{0}=10$ and the searching cost is $C=8$. The other parameters are $\triangle q=q_{i+1}-q_{i}=0.05, A=4, N=54$, and $\mu=0.02$. Given any initial condition, we get the evolution of the distribution of agents. The final optimal
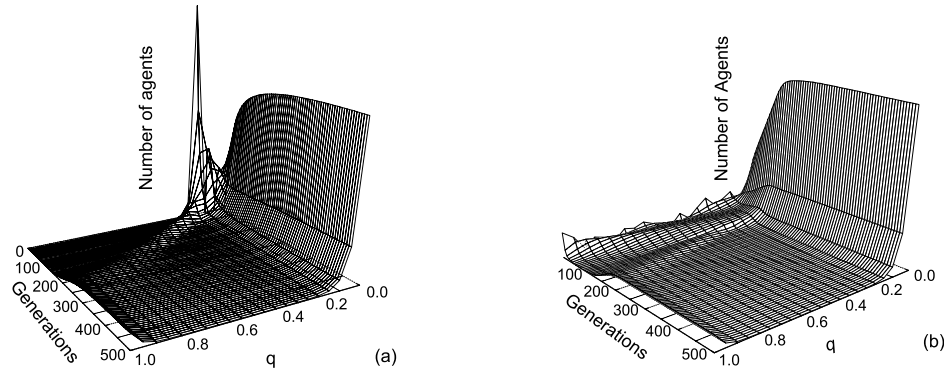

Fig. 3. Evolution from different initial conditions. (a) Homogeneous initial distribution with $q_{k}=0.1$ for every agent. (b) Random initial distribution.

distribution in the Markov chain process is stable and it could be achieved from any given initial conditions (See Figure 3). We have compared the final stable distribution of the Markov chain process with the average distribution in the generations of optimal state in our previous simulation model [20]. As shown in Figure 4, it is notable that the mathematical results are consistent well with the results of simulations. 

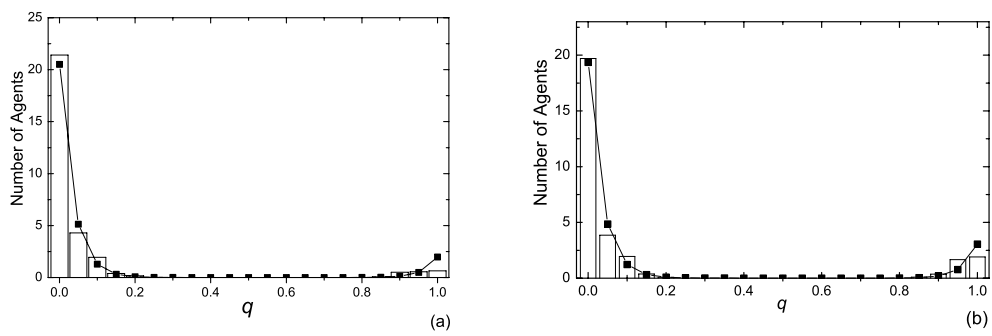

Fig. 4. Comparison of theoretical predictions (solid lines) and simulation results (column bars) of the distributions of agents' number. (a) $P=0.7$ (b) $P=0.4$

\section{Conclusions}

In this paper we have studied the formation of specialization of a simple multiagent model with optimizing evolution behaviors. With a Markov chain model, the multi-agent system has provided discoveries in a concise way to perceive the evolution of labor division. First, specialization is a functional structure in macroscopic level of multi-agent systems. The model has demonstrated how a global structure emerges from the long-term optimizing evolution under the mechanism of increasing returns. Second, an evolutionary process given by transition behavior describes the mechanism of mutation and natural selection. We believe that natural selection may also serve as the basic mechanism in the labor division in social and economic systems. The last but not the least, the results reveal that the stochastic properties in the evolutionary process are necessary to generate macroscopic structure and to reach global optimization.

This work suggests a number of future directions for the study of the optimal behaviors of multi-agent systems. As mentioned in section 2 the model assumes that there is perfect information among agents and the goal of the system is global optimization. These assumptions in fact have suggested that the agents have already formed a colony. It is interesting to study the relationship between individual behavior and global optimization, to see how organization emerges from individual optimum, and to understand how a multi-agent system forms aggregate units.

Acknowledgments. This work was partially supported by the 985 project and NSFC under grant No.70471080 and No.70371072.

\section{References}

1. Theralauz G. Bonabeau E. and Deneubourg, J., The origin of nest complexity in social insects. Complexity 3 (1998) 15-25.

2. Gordon D., The Organization of work in social insect colonies. Complexity 8 (2003) 43-46. 
3. Robinson G., Regulation of division of labor in insect societies. Annu. Rev. Entomol 37 (1992) 637-665.

4. Bonabeau E., Theraulaz, G. and Deneubourg J., Quantitative study of the fixed threshold model for the regulation of division of labour in insect societies. Proc. Roy. Soc. London B 263 (1996) 1565-1569.

5. Wu J., Di Z., and Yang Z., Division of labor as the result of phase transition. Phasica A 323, (2003) 663-676.

6. Zimmermann G., Neuneier R. and Grothmann R., Multi-Agent market modeling of foreign exchange rates. Advances in Complex Systems 4 (2001) 29-43.

7. Darbyshire, P., Effects of communication on group learning rates in a multi-agent environment. Advances in Complex Systems 6 (2003) 405-426.

8. Juanico, D., Monterola, C. and Saloma, C., Cluster formation by allelomimesis in real-world complex adaptive systems. Phys. Rev. E 71, (2005) 041905.

9. Taylor, P. and Day, T., Behavioural evolultion: cooperate with thy neighbour? Nature 428 (2004) 643-646.

10. Nowak, M., Sasaki, A., Taylor, C. and Fudenberg, D., Emergence of cooperation and evolutionary stability in finite populations. Nature 428 (2004) 646-650.

11. Weiss G. et al. (eds.), Adaption and learning in multi-agent systems. SpringerVerlag, Berlin (1996) 20-35.

12. Kephart J., Hagg T. and Huberman B., Dynamics of computational ecosystems, Phys. Rev. A 40 (1989) 404-421.

13. Hagg T. and Huberman B., Controlling chaos in distributed systems, IEEE Transactions on Systems, Man, and Cybernetics 21 (1991) 1325-1332.

14. Bonabeau E., and G. Theraulaz, Self-organization in social insects, Trend in Ecology and Evolution 12 (1997) 188-193.

15. Holland J., Hidden Order-how adaptation builds complexity. Addison Wesley: MA (1995) 230-300.

16. Holland J. and Miller J., Artificial adaptive agents in economic theory, American Economic Review 81(2) (1991) 365-370.

17. Andriani P., Diversity, Knowledge and complexity theory: some introductory issues. International Journal of Innovation Management 5(2) (2001) 257-274.

18. Savage M., and Askenazi M., Arborscapes: a swarm-based multi-agent ecological disturbance model. Technical Report (STF-TR-98-06-056, Santa Fe Institute, 1998).

19. Fontana W., Buss L., The arrival of the fittest: toward a theory of biological organization, Bull. Math. Biol. 56 (1994) 1-64.

20. Di Z., Chen J., Wang Y., Han Z., Agent division as the results of global optimizing evolution, in; Shi Z., He Q. (eds.), Proceedings of International conferebce on intelligent information technology. Post \& Telecom Press Beijing (2002) 40-46.

21. Arrow K.J., The economic implications of learning by doing, Rev. of Economics Stu. 29 (1962) 155-173. 